\section{Classificação dos periódicos no Sistema Qualis da Capes - A mudança dos critérios é URGENTE!}

Editores científicos de revistas médicas brasileiras*

$\mathrm{A}^{\mathrm{s}}$ Associação Médica Brasileira (AMB) preocupada com o futuro das publicações científicas brasileiras, depois da divulgação dos novos critérios Qualis da Capes, organizou uma série de encontros em sua sede em São Paulo. Os Editores das principais revistas médicas do país, diretores da Associação Brasileira de Editores Científicos (Abec) e os coordenadores das áreas de Medicina II e Medicina III da Capes trocaram informações e, acima de tudo, elaboraram propostas para aprimorar o processo de avaliação dos periódicos científicos brasileiros pelo novo sistema Qualis da Capes. A produção científica classificada pelo Qualis constituirá um dos itens principais da avaliação dos programas de pós-graduação no último triênio. Considerando que a principal fonte de artigos científicos para as revistas brasileiras são provenientes dos programas de pós-graduação vinculados à Capes, era muito importante afinar o discurso e garantir uma linguagem comum para todas as partes envolvidas. Do lado dos Editores há receio de que os novos critérios da Capes possam criar uma subclasse de periódicos baseada exclusivamente no Fator de Impacto ISI. O critério anterior indicava, como ponto de corte, fator de impacto igual a l. Recentemente, algumas revistas brasileiras conseguiram, com enormes esforços, atingir esse patamar. Entretanto, os novos critérios, além de considerarem apenas o fator de impacto, estabeleceram pontos de corte bem mais elevados. Adotando-se essa medida, as revistas brasileiras passariam a ser preteridas pelos orientadores e alunos de pós-graduação - os grandes produtores da ciência brasileira - criando-se assim um círculo vicioso com o qual seria difícil a sobrevivência dos nossos periódicos.

Pelo lado da Capes, falou o Professor João Pereira Leite, que, além de coordenador da área de Medicina II, é também o atual representante da área de saúde no Conselho Técnico Científico (CTC), órgão máximo da Capes. Este, durante uma de nossas reuniões, fez detalhada explanação sobre os critérios adotados nos triênios anteriores e o impacto destes sobre os Programas de pós-graduação do Brasil. Explicou ainda que, diante da evidente melhoria do nível dos programas, era preciso elevar o ponto de corte ou de separação para melhor discriminá-los e estratificá-los qualitativamente. A partir dos dados provenientes dos programas - colhidos pelo sistema coleta Capes - foi observado que muitos tinham mais de $50 \%$, alguns mais de $80 \%$, de sua produção científica publicada em periódicos dos estratos mais elevados. A Capes, por sua vez, decidiu pela criação de um número maior de estratos para poder reclassificar os periódicos. Foi proposta uma escala decrescente segundo o valor do fator de impacto: Al, A2, B1, B2, B3, B4, B5 e C. Além disso, criou um fator de equivalência segundo o qual a soma de publicações em revistas de estratos inferiores seria equivalente a um número menor de publicações dos estratos superiores. Assim, por exemplo, para uma determinada área, 2 artigos $\mathrm{Bl}$ equivaleriam a 1,2 artigo $\mathrm{Al} ; 1$ artigo $\mathrm{Bl}+1$ artigo $\mathrm{A} 2$ equivaleriam a 1,4 artigo $\mathrm{Al} ; 3$ artigos $\mathrm{B} 2$ equivaleriam a $1,2 \mathrm{Al}$. Segundo o profes-
*Os membros participantes, editores científicos e os nomes das revistas estão listados ao final do artigo

Publicação concomitante nas revistas médicas dos editores científicos participantes do grupo de discussão promovido pelo Departamento Científico da Associação Médica Brasileira (AMB), Brasil 
sor João Leite, "Essa equivalência beneficiaria as revistas com diferentes niveis de qualificação". O professor Leite informou ainda que a nova classificação foi elaborada com base na mediana do fator de impacto das revistas, obtida junto ao Journal Citation Reports (JCR) e calculada anualmente pelo ISI Web of Knowledge. Para o cálculo da mediana, foi elaborada uma lista das revistas em que cada área da Capes publica. De posse dessa lista, bem como dos respectivos fatores de impacto, foi calculada a mediana para cada área e construída a nova estratificação que varia entre $\mathrm{Al}, \mathrm{A} 2, \mathrm{~B} 1$ até $\mathrm{B} 5$ e C.

Os Editores contra-argumentaram o professor Leite lembrando que a característica trienal do processo de avaliação Capes conferiria um relativo descompasso para a reclassificação dos periódicos, a saber: várias revistas brasileiras terão seu fator de impacto aumentado ou publicado pela primeira vez ao longo de 2010, em especial as que acabaram de entrar para o ISI. Além disso, teriam de esperar três anos para mudar de categoria dentro do novo Qualis. Outro questionamento dos Editores diz respeito à escolha do fator de impacto publicado pelo JCR como ÚNICO e universal índice para aferição da qualidade dos periódicos. É grande o desvio-padrão dos valores dos fatores de impacto das revistas. Certamente por isso a Capes utilizou a mediana desses índices para analisar o comportamento da produção dos Programas de pós-graduação. De fato, segundo esse critério, algumas especialidades médicas, como as cirúrgicas, têm suas melhores revistas com fator de impacto mais baixo, o que poderia implicar um viés que lhes seria extremamente desfavorável.

Os dois lados concordaram que a valorização dos periódicos brasileiros é importante para o crescimento e desenvolvimento científico nacional. Para garantir e estimular esse círculo virtuoso, é preciso, entre outros, estimular e fomentar a citação de artigos de autores nacionais, intensificar os esforços de Editores, revisores e autores dos periódicos para aumentar a qualidade dos artigos e, por outro lado, obter maior apoio dos órgãos governamentais, principalmente da Capes e do Conselho Nacional de Desenvolvimento Científico e Tecnológico (CNPq), no que diz respeito, respectivamente, à administração de recursos financeiros e à estratificação qualitativa.

Os resultados dessas discussões foram apresentados em diversos encontros de Editores, coordenadores de Programas de pós-graduação e pesquisadores, sendo complementados por novas sugestões. Que estas ideias, abaixo descritas, sirvam de conclusão para este editorial e que representem, ao mesmo tempo, uma ferramenta importante para a mudança dos critérios de classificação dos periódicos no sistema Qualis da Capes por parte dos organismos responsáveis. As propostas são as seguintes:

- A análise qualitativa dos periódicos brasileiros deve ser reavaliada e não envolver somente o Fator de Impacto publicado pelo Journal Citation Reports (JCR).

- Devem ser consideradas e respeitadas as particularidades de cada área de interesse ou de cada especialidade.

- O parque editorial brasileiro, diferente do restante do mundo que é mantido basicamente pela iniciativa privada, é mantido à custa de Universidades públicas e privadas e associações cientificas de classe.

- Os periódicos brasileiros necessitam de maior apoio e incentivo, que poderão vir na forma de: Bolsa para Editores, apoio financeiro à publicação, maior visibilidade para os periódicos nacionais no exterior, critérios mais objetivos e abrangentes para classificação qualitativa, e apoio diferenciado e correspondente ao desempenho de cada revista.

- Apoio à internacionalização dos periódicos cientificos por meio de suporte para profissionalização do processo editorial e divulgação das revistas em outros países.

- Atualização contínua da classificação dos periódicos junto ao novo Qualis sem precisar esperar pelo prazo da avaliação trienal.

- Participação de representantes de classe (Abec, $A M B$, entre outros) no processo decisório junto ao sistema Qualis da Capes.

- Estímulo vigoroso à citação diretamente na fonte que são os programas de pós-graduação (por exemplo, determinando que Programas de PG notas 6 e 7, além de deverem obrigatoriamente ter uma porcentagem de publicações em revista de alto impacto, devam ter também cotas percentuais de publicações em periódicos nacionais. Com isso estarão contemplados os dois extremos da produção científica, pois os jovens e futuros pesquisadores iniciam sua carreira publicando em periódicos nacionais sob orientação de pesquisadores experientes.

Por fim, para corroborar todas essas ações e preocupada com o desenrolar das repercussões do novo Qualis da Capes e outras avaliações de periódicos, a Abec dedicou, em seu último Encontro Nacional de Editores Científicos, realizado em novembro de 2009, três dias ao Fórum de áreas. Neste, representantes da Capes e Editores de todas as áreas do conhecimento científico discutiram longamente o assunto e ao final propuseram as Diretrizes do Fórum de Áreas do XII Encontro Nacional dos Editores Científicos - 2009, que serão oportunamente enviadas a todas as agências brasileiras de fomento e que deverão ocorrer periodicamente, pois o processo é contínuo. 


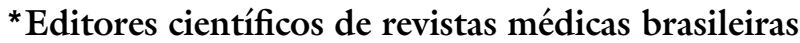

Adagmar Andriolo, Jornal Brasileiro de Patologia e Medicina Laboratorial; Aécio Flávio Meireles Souza, Revista GED; Alberto Queiroz Farias, Revista Arquivos de Gastroenterologia; Alfredo José Afonso Barbosa, Jornal Brasileiro de Patologia e Medicina Laboratorial; Antonio Spina França Netto, Revista Arquivos de Neuro-Psiquiatria; Arnaldo José Hernandez, Revista Brasileira de Medicina do Esporte; Aroldo F. Camargos, Revista Femina; Benedito Barraviera, Journal of Venomous Animals and Toxins including Tropical Diseases; Bogdana Victoria Kadunc, Surgical of Cosmetic Dermatology da Sociedade Brasileira de Dermatologia; Bruno Caramelli, Revista da Associação Médica Brasileira; Carlos Eduardo Aguilera Campos, Revista Brasileira de Medicina de Familia $e$ Comunidade; Carlos Brites, Brazilian Journal of Infectious Diseases; Dejair Caitano do Nascimento, Hansenologia Internationalis; Domingo M. Braile, Revista Brasileira de Cirurgia Cardiovascular; Dov Charles Goldenberg, Revista Brasileira de Cirurgia Plástica; Edmund Chada Baracat, Revista da Associação Médica Brasileira, Edna T. Kimura, Arquivos Brasileiros de Endocrinologia e Metabologia; Edson Marchiori, Revista Radiologia Brasileira, Eduardo de Paula Vieira, Revista Brasileira de Coloproctologia; Eros Antônio de Almeida, Revista da Sociedade Brasileira de Clínica Médica; Geraldo Pereira Jotz, Revista Brasileira de Cirurgia Cabeça e Pescoço; Gilberto Camanho, Revista Brasileira de Ortopedia; Gilberto Friedman, Revista Brasileira de Terapia Intensiva; Giovanni Guido Cerri, Radiologia Brasileira; Ivomar Gomes Duarte, Revista de Administração em Saúde; Izelda Maria Carvalho Costa, Anais Brasileiros de Dermatologia; João Ferreira de Mello Júnior; Brazilian Journal of Otorbinolaryngology; Joel Faintuch, Revista Brasileira de Nutrição Clínica; José Antônio Baddini Martinez, Jornal Brasileiro de Pneumologia; José Antonio Livramento, Revista Arquivos de Neuropsiquiatria; José Eduardo Ferreira Manso, Revista do Colégio Brasileiro de Cirurgiões; José Luiz Gomes do Amaral, Revista da Associação Médica Brasileira; Linamara Rizzo Battistella; Revista Acta Fisiátrica; Luís dos Ramos Machado, Revista Arquivos de Neuropsiquiatria; Luiz Felipe P. Moreira, Arquivos Brasileiros de Cardiologia; Luiz Henrique Gebrim, Revista Brasileira de Mastologia; Marcelo Madeira; Revista Brasileira de Mastologia; Marcelo Riberto, Revista Acta Fisiátrica; Marcus Bastos, Jornal Brasileiro de Nefrologia; Mário Cícero Falcão, Revista Brasileira de Nutrição Clínica; Mario J. da Conceição, Revista da Sociedade Brasileira de Anestesiologia; Mauricio Rocha e Silva, Revista Clinics; Milton Artur Ruiz, Revista Brasileira de Hematologia e Hemoterapia; Milton K. Shibata, Arquivos Brasileiros de Neurocirurgia; Mittermayer Barreto Santiago, Revista Brasileira de Reumatologia; Nelson Adami Andreollo, Arquivos Brasileiros de Cirurgia Digestiva; Osvaldo Malafaia, Arquivos Brasileiros de Cirurgia Digestiva; Regina Helena Garcia Martins, Brazilian Journal of Otorhinolaryngology; Renato Soibelmann Procianoy, Jornal de Pediatria; Ricardo Baroudi, Revista Brasileira de Cirurgia Plástica; Ricardo Fuller, Revista Brasileira de Reumatologia; Ricardo Guilherme Viebig, Arquivos de Gastroenterologia; Ricardo Nitrini, Dementia \& Neuropsychologia; Rita Cristina Mainieri R. de Moura, Revista da Associação Brasileira de Medicina de Tráfego; Rogério Dedivitis, Revista Brasileira de Cirurgia Cabeça e Pescoço; Ronaldo Damião, Urologia Contemporânea; Sergio Lianza, Revista Medicina de Reabilitação; Sigmar de Mello Rode, Brazilian Oral Research; Winston Bonetti Yoshida, Jornal Vascular Brasileiro; Zuher Handar, Revista Brasileira de Medicina do Trabalbo. 